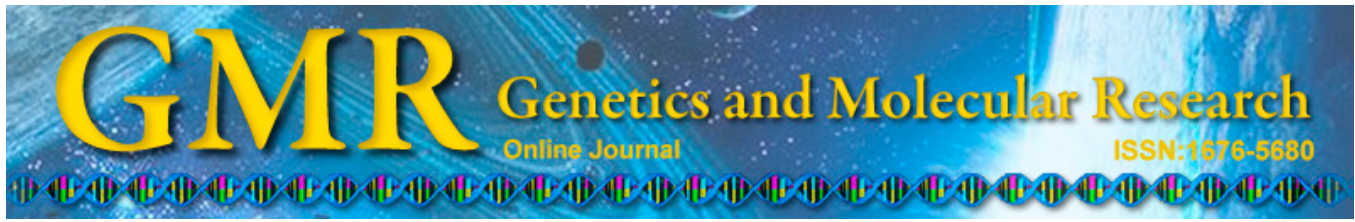

\title{
Significant interaction of $A P O E$ rs4420638 polymorphism with HDL-C and APOA-I levels in coronary heart disease in Han Chinese men
}

\author{
Y. Huang', H.D. Ye' ${ }^{2}$ X. Gao', S. Nie', Q.X. Hong ${ }^{2}$, H.H. Ji², J. Sun', \\ ${ }^{1}$ Department of Neurosurgery, Ningbo First Hospital, \\ Ningbo Hospital of Zhejiang University, Ningbo, Zhejiang, China \\ ${ }^{2}$ Zhejiang Provincial Key Laboratory of Pathophysiology, \\ School of Medicine, Ningbo University, Ningbo, Zhejiang, China \\ Neuropsychiatric Disorders (Ministry of Education), \\ Shanghai Jiao Tong University, Shanghai, China \\ Corresponding authors: S.W. Duan / X. Gao \\ E-mail: duanshiwei@nbu.edu.cn / qinyuecui@163.com
} S.J. Zhou ${ }^{1}$, B. Fei ${ }^{1}$, K.Q. Li ${ }^{1}$, J.K. Zhao ${ }^{1}$, Z.P. Wang ${ }^{1}$, M.Q. Xu ${ }^{3}$ and S.W. Duan ${ }^{2}$ ${ }^{3}$ Bio-X Institutes, Key Laboratory for the Genetics of Developmental and

Genet. Mol. Res. 14 (4): 13414-13424 (2015)

Received June 20, 2015

Accepted September 8, 2015

Published October 28, 2015

DOI http://dx.doi.org/10.4238/2015.October.28.3

\begin{abstract}
Apolipoprotein E (APOE) is recognized for its importance in lipoprotein metabolism and cardiovascular disease. We evaluated the association between APOE rs4420638 genotypes and circulating lipid concentrations along with the risk of coronary heart disease (CHD). We conducted a case-control study involving 1508 individuals to investigate the contribution of rs4420638 to the risk of CHD in Han Chinese. In addition, we performed a meta-analysis to evaluate the association between rs4420638 and CHD in Europeans and Asians. The results show that rs4420638 is significantly correlated with increased CHD risk in male Han Chinese [P $=0.040$, odds ratio (OR) $=1.34,95 \%$ confidential interval $(95 \% \mathrm{CI})=1.01-1.78]$ and is likely to increase the risk of CHD under the dominant model in males $(\mathrm{P}=$
\end{abstract}


$0.036, \mathrm{OR}=1.38,95 \% \mathrm{CI}=1.02-1.88$ ). A further subgroup analysis by rs4420638 genotype found a significant association of rs4420638 AA with high-density lipoprotein cholesterol (HDL-C) $(\mathrm{P}=0.012)$ and APOA-I levels $(\mathrm{P}=0.0001)$ in males. The meta-analysis suggests that rs4420638 significantly increases the risk of $\mathrm{CHD}(\mathrm{OR}=1.18,95 \% \mathrm{CI}$ $=1.14-1.22, \mathrm{P}<0.0001$, fixed-effect method). Our case-control study shows that rs4420638 genotype AA has a significant association with the concentrations of circulating HDL-C and APOA-I in CHD in Han Chinese males. The meta-analysis suggests that rs4420638 is associated with CHD risk in Europeans and Asians.

Key words: Coronary heart disease; High-density lipoprotein cholesterol; HDL-C; APOE; APOA-I; Polymorphism

\section{INTRODUCTION}

It is widely accepted that coronary heart disease (CHD) is a major public health problem worldwide (Graff-Iversen et al., 2014). CHD is a complex disease and is influenced by a combination of genetic and environmental factors (Puddu et al., 2014). Despite extensive efforts in the identification of genetic factors that affect susceptibility to CHD (Roberts and Stewart, 2012), the underlying mechanisms remain unclear.

Circulating blood lipid concentration is a strong independent risk factor for CHD (Rached et al., 2014). Total cholesterol (TC) and lipoproteins, including apolipoproteins A, $\mathrm{B}$, and $\mathrm{E}$ (APOA, APOB, and APOE), may play an important role in the developmental and physiological processes of CHD. Research has shown that high levels of low-density lipoprotein cholesterol (LDL-C) and TC lead to atherosclerosis and CHD (Isley, 2006), and that high-density lipoprotein cholesterol (HDL-C) levels are negatively correlated to CHD (Subedi et al., 2014).

APOE [Online Mendelian Inheritance in Man (OMIM) No. 107741], which encodes APOE protein, has the chromosomal locus 19q13.32 and consists of four exons and three introns. It is a member of the LDL receptor gene family and exists in a cluster with apolipoprotein C-I and C-II genes (APOC1 and APOC2) (Onat et al., 2013). Defects in APOE can cause an increase in the levels of plasma cholesterol and triglycerides (TGs) due to impaired clearance of chylomicron, very low-density lipoprotein, and LDL-C remnants (Mendivil et al., 2013). APOE is recognized for its importance in lipoprotein metabolism and cardiovascular disease (Onat et al., 2013). APOE polymorphisms (rs429358 and rs7412) have shown an association with serum lipids in certain populations (Smalinskiene et al., 2013). These variants are associated with differences in lipid risk factors and atherosclerosis (Heidari et al., 2013). APOE polymorphisms have been extensively studied as predictors of cardiovascular disease (Smalinskiene et al., 2013). The single nucleotide polymorphism (SNP) rs4420638 is located in the APOE-C1-C2 cluster on the $19 \mathrm{q} 13$ chromosome. It has been shown to be in high linkage disequilibrium with $\mathrm{rs} 429358\left(\mathrm{r}^{2}>0.7\right)$ and to be significantly associated with the increased risk of CHD (Elliott et al., 2009). SNP rs4420638 is associated with the modulation of many lipids in humans (Grallert et al., 2012).

In this study, we recruited 776 CHD patients and 732 controls from Zhejiang in Eastern China and performed a case-control study to investigate the contribution of rs4420638 
polymorphism to the risk of CHD in the Han Chinese population. We also examined the association of the genotypes with circulating lipid concentrations. In addition, we performed a meta-analysis to evaluate the association between rs4420638 and CHD risk in Europeans and Asians.

\section{MATERIAL AND METHODS}

\section{Sample collection}

The study was approved by the Ethics Committee of the School of Medicine, Ningbo University. All 1508 unrelated inpatients were recruited from the Affiliated Hospitals of Ningbo University (Ningbo Lihuili Hospital, Ningbo No. 3 Hospital and Ningbo Yinzhou Hospital) and the Second Affiliated Hospital of Zhejiang University between May 2008 and April 2013. All volunteers were defined through the standardized coronary angiography protocol (Higgs et al., 2005) and the results were assessed by two or three individual physicians. Among these, 776 CHD patients (533 males and 243 females, mean age: $62.4 \pm 0.3$ years) were diagnosed by angiographic evidence, including coronary arteries with $50 \%$ or greater stenosis, or a history of prior angioplasty or coronary artery bypass surgery. The 732 controls (419 males and 313 females, mean age: $59.7 \pm 0.4$ years) were patients that had less than $50 \%$ occlusion of any coronary vessel in the angiographic results and no history of atherosclerotic vascular disease. All individuals were Han Chinese, originating from Zhejiang Province and without congenital heart disease, cardiomyopathy, or severe liver or kidney disease.

\section{Biochemical variables}

Blood samples were collected in $3.2 \%$ sodium citrate-treated tubes and stored at $4{ }^{\circ} \mathrm{C}$ for short periods or at $-80^{\circ} \mathrm{C}$ for long periods. Levels of plasma TG, serum TC, HDL-C, and LDL-C were measured using an enzymatic end point assay (Lopes-Virella et al., 1977). The APOA-I, APOB, and APOE levels were measured using the transmission turbidimetry method (Jiang et al., 2013).

\section{Genotyping}

Genomic DNA was isolated from peripheral blood samples using a nucleic acid extraction automatic analyzer (Lab-Aid 820, Xiamen, China) and all the DNA samples were stored in TE buffer (TE is Tris and ethylenediaminetetraacetic acid), at $-80^{\circ} \mathrm{C}$. The polymerase chain reaction (PCR) primers for rs4420638 were as follows: forward, 5'-ACGTTGGATGATTG AACCCTCAGCCTAGCA-3'; reverse, 5'-ACGTTGGATGAAAAACCTCAGCCCCTCATC -3'; extension primer, 5'-GCAGCTCGGGTAGACCACAC-3'. The PCR amplification was performed on an ABI GeneAmp ${ }^{\circledR}$ PCR System 9700 (dual 384-well blocks) (Applied Biosystems, Foster City, CA, USA). Primer extension for genotyping was performed on a MassARRAY iPLEX ${ }^{\circledR}$ assay platform (Sequenom, San Diego, CA, USA) according to the manufacturer instructions. The PCR process included an initial denaturation at $94^{\circ} \mathrm{C}$ for $15 \mathrm{~s}$, followed by 45 cycles at $94^{\circ} \mathrm{C}$ for $20 \mathrm{~s}, 56^{\circ} \mathrm{C}$ for $30 \mathrm{~s}, 72^{\circ} \mathrm{C}$ for $1 \mathrm{~min}$, and then a final extension at $72^{\circ} \mathrm{C}$ for $1 \mathrm{~min}$. Quality controls were described in our previous study (Huang et al., 2013). 


\section{Retrieval of published studies and data extraction}

A literature search was performed for the meta-analysis up to April 17, 2014, in electronic databases including PubMed, Web of Science, and China National Knowledge Infrastructure (CNKI). We used the following Medical Subject heading (MeSH) terms for searching: "polymorphism"; "ApoE"; "ApoC1"; and "rs4420638" paired with "coronary heart disease" or "coronary artery disease". We also read the full text to find relevant information. References listed in the retrieved articles, review articles, or previous meta-analyses were searched to appraise other studies for potential relevance. Data were extracted by two authors and divergence was resolved by consensus. The information collected from each study comprised the author, publication year, number of cases and controls, OR, and 95\% CI.

\section{Statistical analyses}

In the biochemical analysis between CHD cases and controls, continuous variables were described by mean and standard deviation (SD) using the Student $t$-test. For the nonparametric indicators, the Kruskal-Wallis test was applied. All $\mathrm{P}$ values were adjusted for age and gender in the comparison of lipid levels. Hardy-Weinberg equilibrium was analyzed by the Arlequin program (version 3.5; Bern, Switzerland). Comparison of the genotype distribution and allele frequencies between cases and controls was calculated using the CULMP16 software according to the method described previously (Huang et al., 2013). Power analysis of the case-control study was performed using the Power and Sample Size Calculation software (version 3.0.43; Nashville, TN, USA). ORs and 95\%CIs were all estimated by chi-squared analyses using the SPSS 18.0 statistic software. A two-tailed $\mathrm{P}$ value $<0.05$ was considered to be statistically significant.

The meta-analysis was performed using the Stata statistical software (Version 11; StataCorp. LP, College Station, TX, USA). Heterogeneity of the studies was calculated by the inconsistency index $\left(\mathrm{I}^{2}\right)$ test at a significance level of 50\%. The random-effect model was used to calculate the combined ORs for the studies with strong heterogeneity $\left(\mathrm{I}^{2}>50 \%\right)$. Otherwise, the fixed-effect model was used (Lian et al., 2013). Publication bias was determined by funnel plots.

\section{RESULTS}

The genotypes and allele frequencies of the rs 4420638 polymorphism in the case and control groups are shown in Table 1. The distributions of genotypes were all within HardyWeinberg equilibrium $(\mathrm{P}>0.05)$. No association was found between rs4420638 and CHD $\left(\chi^{2}\right.$ $=2.82, \mathrm{P}=0.931, \mathrm{OR}=1.21,95 \% \mathrm{CI}=0.97-1.50)$. However, a breakdown analysis by gender indicated a significant association in males $\left(\chi^{2}=4.20, \mathrm{P}=0.040, \mathrm{OR}=1.34,95 \% \mathrm{CI}=1.01\right.$ $1.78)$, in contrast with a lack of association in females $\left(\chi^{2}=0.01, \mathrm{P}=0.920, \mathrm{OR}=1.02,95 \% \mathrm{CI}\right.$ $=0.71-1.46)$.

Significantly increased risk was observed between rs4420638 and CHD under the dominant model in males (Table 2; AA versus $\mathrm{AG}+\mathrm{GG}, \chi^{2}=4.37, \mathrm{P}=0.036, \mathrm{OR}=1.38$, $95 \% \mathrm{CI}=1.02-1.88)$. No significant results were found in the alternative genetic models and subgroup analyses by gender and age (Tables 2 and $3 ; \mathrm{P}>0.05$ ). 
Table 1. Genotype and allele distributions of rs4420638.

\begin{tabular}{|c|c|c|c|c|c|c|c|c|c|c|c|}
\hline \multirow[t]{2}{*}{ Gender } & \multirow[t]{2}{*}{ Groups (N) } & \multicolumn{3}{|c|}{ Genotype (N) } & \multirow[t]{2}{*}{$\chi^{2}$} & \multirow{2}{*}{$P($ d.f. = 2) } & \multirow{2}{*}{$\frac{\text { Protective allele }}{\mathrm{A}(\%)}$} & \multirow{2}{*}{$\frac{\text { Risk allele }}{G(\%)}$} & \multirow[t]{2}{*}{$\chi^{2}$} & \multirow[t]{2}{*}{$\mathrm{P}(\mathrm{d} . \mathrm{f} .=1)$} & \multirow[t]{2}{*}{ OR $(95 \% \mathrm{CI})$} \\
\hline & & AA (\%) & AG (\%) & GG (\%) & & & & & & & \\
\hline All & $\begin{array}{l}\text { Cases (776) } \\
\text { Controls (732) }\end{array}$ & $\begin{array}{l}582(75.0) \\
577(78.8)\end{array}$ & $\begin{array}{l}183(23.6) \\
146(19.9)\end{array}$ & $\begin{array}{r}11(1.4) \\
9(1.2)\end{array}$ & 3.10 & 0.21 & $\begin{array}{l}1347(86.8) \\
1300(88.8)\end{array}$ & $\begin{array}{l}205(13.2) \\
164(11.2)\end{array}$ & 2.82 & 0.931 & $\begin{array}{c}1.21 \\
(0.97-1.50)\end{array}$ \\
\hline Male & $\begin{array}{l}\text { Cases (533) } \\
\text { Controls (419) }\end{array}$ & $\begin{array}{l}394(73.9) \\
334(79.7)\end{array}$ & $\begin{array}{r}133(25.0) \\
82(19.6)\end{array}$ & $\begin{array}{l}6(1.1) \\
3(0.7)\end{array}$ & 4.45 & 0.11 & $\begin{array}{l}921(86.4) \\
750(89.5)\end{array}$ & $\begin{array}{r}145(13.6) \\
88(10.5)\end{array}$ & 4.20 & 0.040 & $\begin{array}{c}1.34 \\
(1.01-1.78)\end{array}$ \\
\hline Female & $\begin{array}{l}\text { Cases (243) } \\
\text { Controls (313) }\end{array}$ & $\begin{array}{l}188(77.4) \\
243(77.6)\end{array}$ & $\begin{array}{l}50(20.6) \\
64(20.4)\end{array}$ & $\begin{array}{l}5(2.1) \\
6(1.9)\end{array}$ & 0.02 & 0.99 & $\begin{array}{l}426(87.7) \\
550(87.9)\end{array}$ & $\begin{array}{l}60(12.4) \\
76(12.1)\end{array}$ & 0.01 & 0.920 & $\begin{array}{c}1.02 \\
(0.71-1.46)\end{array}$ \\
\hline
\end{tabular}

Results with $\mathrm{P}$ values less than 0.05 are in bold font.

Table 2. Genetic testing under the dominant and recessive models.

\begin{tabular}{|c|c|c|c|c|c|c|c|c|c|c|c|}
\hline \multirow[t]{2}{*}{ Gender } & \multirow{2}{*}{$\begin{array}{l}\text { Groups }(\mathrm{N}) \\
\text { rs4420638 }\end{array}$} & \multicolumn{2}{|c|}{ Dominant } & \multirow[t]{2}{*}{$\chi^{2}$} & \multirow[t]{2}{*}{$P($ d.f. $=1)$} & \multirow[t]{2}{*}{ OR $(95 \% \mathrm{CI})$} & \multicolumn{2}{|c|}{ Recessive } & \multirow[t]{2}{*}{$\chi^{2}$} & \multirow[t]{2}{*}{$P($ d.f. $=1)$} & \multirow[t]{2}{*}{ OR $(95 \% \mathrm{CI})$} \\
\hline & & $\mathrm{AA}$ & $\mathrm{AG}+\mathrm{GG}$ & & & & $\mathrm{AA}+\mathrm{AG}$ & GG & & & \\
\hline \multirow[t]{2}{*}{ All } & Cases (776) & 582 & 194 & \multirow[t]{2}{*}{3.10} & \multirow[t]{2}{*}{0.078} & 1.24 & 765 & 11 & \multirow[t]{2}{*}{0.10} & \multirow[t]{2}{*}{0.750} & 1.16 \\
\hline & Controls (732) & 577 & 155 & & & $(0.98-1.58)$ & 723 & 9 & & & $(0.48-2.80)$ \\
\hline \multirow[t]{2}{*}{ Male } & Cases (533) & 394 & 139 & \multirow[t]{2}{*}{4.37} & \multirow[t]{2}{*}{0.036} & 1.38 & 527 & 6 & \multirow[t]{2}{*}{ NA } & \multirow[t]{2}{*}{ NA } & 1.58 \\
\hline & Controls (419) & 334 & 85 & & & (1.02-1.88) & 416 & 3 & & & $(0.39-6.35)$ \\
\hline \multirow[t]{2}{*}{ Female } & Cases (243) & 188 & 55 & \multirow[t]{2}{*}{0.01} & \multirow[t]{2}{*}{0.920} & 1.02 & 238 & 5 & \multirow[t]{2}{*}{ NA } & \multirow[t]{2}{*}{ NA } & 1.07 \\
\hline & Controls (313) & 243 & 70 & & & $(0.68-1.52)$ & 307 & 6 & & & $(0.32-3.56)$ \\
\hline
\end{tabular}

Results with P values less than 0.05 are in bold font.

Table 3. Breakdown association test in different age subgroups.

\begin{tabular}{|c|c|c|c|c|c|c|c|c|c|c|c|c|}
\hline \multirow[t]{2}{*}{ Gender } & \multirow{2}{*}{$\begin{array}{c}\text { Age } \\
\text { (years) }\end{array}$} & \multirow{2}{*}{$\begin{array}{l}\text { Groups }(\mathrm{N}) \\
\text { rs } 4420638\end{array}$} & \multicolumn{3}{|c|}{ Genotype (N) } & \multirow[t]{2}{*}{$\chi^{2}$} & \multirow[t]{2}{*}{$P($ d.f. $=2)$} & \multicolumn{2}{|c|}{ Allele $(\mathrm{N})$} & \multirow[t]{2}{*}{$\chi^{2}$} & \multirow[t]{2}{*}{$P($ d.f. $=1)$} & \multirow[t]{2}{*}{ OR $(95 \% \mathrm{CI})$} \\
\hline & & & AA & $\mathrm{AG}$ & GG & & & A & G & & & \\
\hline \multirow[t]{4}{*}{ All } & \multirow[t]{2}{*}{$<65$} & Cases (448) & 343 & 100 & 5 & \multirow[t]{2}{*}{1.37} & \multirow[t]{2}{*}{0.51} & 786 & 110 & \multirow[t]{2}{*}{1.32} & \multirow[t]{2}{*}{0.250} & \multirow[t]{2}{*}{$1.18(0.89-1.57)$} \\
\hline & & Controls (500) & 398 & 98 & 4 & & & 894 & 106 & & & \\
\hline & \multirow[t]{2}{*}{$\geq 65$} & Cases (328) & 239 & 83 & 6 & \multirow[t]{2}{*}{1.65} & \multirow[t]{2}{*}{0.44} & 561 & 95 & \multirow[t]{2}{*}{0.90} & \multirow[t]{2}{*}{0.343} & \multirow[t]{2}{*}{$1.18(0.83-1.68)$} \\
\hline & & Controls (232) & 179 & 48 & 5 & & & 406 & 58 & & & \\
\hline \multirow[t]{4}{*}{ Male } & \multirow[t]{2}{*}{$<65$} & Cases (320) & 246 & 72 & 2 & \multirow[t]{2}{*}{1.50} & \multirow[t]{2}{*}{0.47} & 564 & 76 & \multirow[t]{2}{*}{1.37} & \multirow[t]{2}{*}{0.242} & \multirow[t]{2}{*}{$1.24(0.86-1.78)$} \\
\hline & & Controls (296) & 239 & 56 & 1 & & & 534 & 58 & & & \\
\hline & \multirow[t]{2}{*}{$\geq 65$} & Cases (213) & 148 & 61 & 4 & \multirow[t]{2}{*}{2.37} & \multirow[t]{2}{*}{0.31} & 357 & 69 & \multirow[t]{2}{*}{1.99} & \multirow[t]{2}{*}{0.162} & \multirow[t]{2}{*}{$1.39(0.88-2.21)$} \\
\hline & & Controls (123) & 95 & 26 & 2 & & & 216 & 30 & & & \\
\hline Female & $<65$ & Cases (128) & 97 & 28 & 3 & 0.44 & 0.80 & 222 & 34 & 0.33 & 0.574 & $1.15(0.72-1.84)$ \\
\hline & & Controls (204) & 159 & 42 & 3 & & & 360 & 48 & & & \\
\hline & $\geq 65$ & Cases (115) & 91 & 22 & 2 & 0.32 & 0.85 & 204 & 26 & 0.25 & 0.623 & $0.86(0.49-1.53)$ \\
\hline & & Controls (109) & 84 & 22 & 3 & & & 190 & 28 & & & \\
\hline
\end{tabular}

It is well known that the $A P O E$ variant is a predictor of plasma lipid levels in cardiovascular disease (Yin et al., 2013). Therefore, we further stratified the data analysis into different lipids by rs4420638 genotype. As shown in Table 4 and Figure 1, there were significantly higher levels of HDL-C $(\mathrm{P}=0.014)$ and APOA-I $(\mathrm{P}<0.0001)$ in controls than in cases in the rs4420638-AA carriers. In a further breakdown analysis by gender, we observed similar significant associations between HDL-C $(\mathrm{P}=0.012$; Table 5$)$ and APOA-I levels $(\mathrm{P}=0.0001$; Figure 1) with CHD in the male rs4420638-AA carriers. However, no evidence of association was found in the female subgroup analysis (data not shown). 
Table 4. Comparison of lipid levels between cases and controls under the dominant model.

\begin{tabular}{|c|c|c|c|c|c|c|}
\hline \multirow[t]{2}{*}{ Character } & \multicolumn{3}{|c|}{ AA } & \multicolumn{3}{|c|}{$\mathrm{AG}+\mathrm{GG}$} \\
\hline & Case & Control & $\mathrm{P}$ & Case & Control & $P$ value \\
\hline Age (years) & $62.48 \pm 9.19$ & $59.62 \pm 10.95$ & $<0.0001$ & $62.16 \pm 10.04$ & $60.06 \pm 9.35$ & 0.046 \\
\hline Gender (male) & 394 & 334 & 0.098 & 139 & 85 & 0.126 \\
\hline LDL-C (mM) & $2.55 \pm 0.93$ & $2.58 \pm 0.85$ & 0.670 & $2.63 \pm 1.01$ & $2.62 \pm 0.89$ & 0.458 \\
\hline $\mathrm{TC}(\mathrm{mM})$ & $4.32 \pm 1.15$ & $4.40 \pm 1.06$ & 0.944 & $4.44 \pm 1.20$ & $4.44 \pm 0.99$ & 0.377 \\
\hline HDL-C (mM) & $1.09 \pm 0.27$ & $1.14 \pm 0.27$ & 0.014 & $1.08 \pm 0.28$ & $1.15 \pm 0.33$ & 0.204 \\
\hline $\mathrm{TG}(\mathrm{mM})$ & $1.67 \pm 1.04$ & $1.62 \pm 0.99$ & 0.246 & $1.78 \pm 1.25$ & $1.57 \pm 0.89$ & 0.071 \\
\hline APOA-I (mg/dL) & $0.99 \pm 0.22$ & $1.05 \pm 0.21$ & $<0.0001$ & $1.01 \pm 0.24$ & $1.01 \pm 0.23$ & 0.646 \\
\hline $\mathrm{APOB}(\mathrm{mg} / \mathrm{dL})$ & $0.78 \pm 0.27$ & $0.81 \pm 0.26$ & 0.138 & $0.84 \pm 0.29$ & $0.81 \pm 0.26$ & 0.195 \\
\hline $\operatorname{APOE}(\mathrm{mg} / \mathrm{dL})$ & $4.37 \pm 1.72$ & $4.43 \pm 1.85$ & 0.890 & $4.29 \pm 1.62$ & $4.13 \pm 1.67$ & 0.175 \\
\hline
\end{tabular}

Results with $\mathrm{P}$ values less than 0.05 are in bold font. $\mathrm{P}$ values were adjusted for age and gender. LDL-C $=$ low-density lipoprotein cholesterol; TC $=$ total cholesterol; HDL-C $=$ high-density lipoprotein cholesterol; $\mathrm{TG}=$ triglycerides.
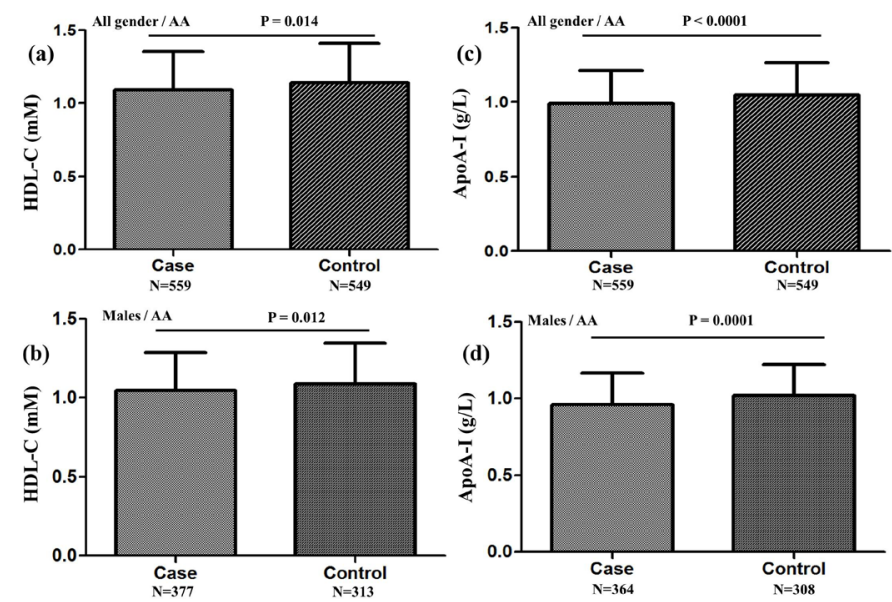

Figure 1. rs4420638 genotype AA shows significant association with HDL-C and APOA-I levels in CHD patients. (a) Comparison of HDL-C levels between cases and controls according to the genotype AA; (b) Comparison of HDL-C level in males between cases and controls according to the genotype AA; (c) Comparison of APOA-I levels in males between cases and controls according to the genotype AA; (d) Comparison of APOA-I levels between cases and controls according to the genotype AA.

Table 5. Comparison of lipid levels in gender subgroups under the dominant model.

\begin{tabular}{|c|c|c|c|c|c|c|}
\hline \multirow[t]{2}{*}{ Character } & \multicolumn{3}{|c|}{ AA } & \multicolumn{3}{|c|}{$\mathrm{AG}+\mathrm{GG}$} \\
\hline & Case & Control & $P$ value & Case & Control & $P$ value \\
\hline \multicolumn{7}{|l|}{ Male } \\
\hline Age (years) & $61.65 \pm 9.34$ & $58.12 \pm 11.25$ & $<0.0001$ & $61.90 \pm 10.60$ & $58.98 \pm 9.60$ & 0.039 \\
\hline LDL-C (mM) & $2.46 \pm 0.91$ & $2.46 \pm 0.83$ & 0.592 & $2.55 \pm 1.02$ & $2.53 \pm 0.86$ & 0.669 \\
\hline $\mathrm{TC}(\mathrm{mM})$ & $4.15 \pm 1.10$ & $4.25 \pm 1.03$ & 0.631 & $4.30 \pm 1.21$ & $4.30 \pm 0.90$ & 0.722 \\
\hline HDL-C (mM) & $1.05 \pm 0.24$ & $1.09 \pm 0.26$ & 0.012 & $1.05 \pm 0.29$ & $1.10 \pm 0.33$ & 0.362 \\
\hline $\mathrm{TG}(\mathrm{mM})$ & $1.65 \pm 1.03$ & $1.70 \pm 1.11$ & 0.833 & $1.75 \pm 1.24$ & $1.63 \pm 0.96$ & 0.307 \\
\hline ApoA-I (mg/dL) & $0.96 \pm 0.21$ & $1.02 \pm 0.20$ & 0.0001 & $0.98 \pm 0.24$ & $0.99 \pm 0.23$ & 0.865 \\
\hline $\mathrm{ApoB}(\mathrm{mg} / \mathrm{dL})$ & $0.76 \pm 0.26$ & $0.79 \pm 0.26$ & 0.218 & $0.81 \pm 0.29$ & $0.82 \pm 0.27$ & 0.908 \\
\hline ApoE (mg/dL) & $4.10 \pm 1.55$ & $4.36 \pm 2.09$ & 0.232 & $4.08 \pm 1.54$ & $3.83 \pm 1.72$ & 0.264 \\
\hline
\end{tabular}

Results with $\mathrm{P}$ values less than 0.05 are in bold font. $\mathrm{P}$ values were adjusted for age. LDL-C $=$ low-density lipoprotein cholesterol; $\mathrm{TC}=$ total cholesterol; HDL-C $=$ high-density lipoprotein cholesterol; $\mathrm{TG}=$ triglycerides. 
As shown in Figure 2, our meta-analysis involved 18 study stages (Clarke et al., 2006; Farrall et al., 2006; Helgadottir et al., 2007; Hofman et al., 2007; Samani et al., 2007; Schunkert et al., 2008; Stirnadel et al., 2008; Elliott et al., 2009; Erdmann et al., 2009; Myocardial Infarction Genetics Consortium et al., 2009; Marques-Vidal et al., 2009; Stewart et al., 2009; Waterworth et al., 2010; Ken-Dror et al., 2010) among 20,498 cases and 52,405 controls from European and Asian ethnic backgrounds. No significant heterogeneity was found among the enrolled studies $\left(\mathrm{I}^{2}\right.$ $=30.6 \%, \mathrm{P}=0.107)$. The pooled ORs suggest a strong protective effect of rs 4420638 against CHD risk $(\mathrm{OR}=1.18,95 \% \mathrm{CI}=1.14-1.22, \mathrm{P}<0.0001$, fixed-effect method $)$. Funnel plots were used to assess publication bias in the studies. As shown in Figure 3, the results do not show any evidence of publication bias $\left(\mathrm{P}_{\mathrm{Begg}}=0.13, \mathrm{P}_{\text {Egger }}=0.36\right)$. A further subgroup analysis by ethnicity showed that rs 4420638 was significantly associated with $\mathrm{CHD}$ risk in both Europeans $(\mathrm{OR}=1.18,95 \% \mathrm{CI}$ $=1.13-1.22, \mathrm{P}<0.0001)$ and Asians $(\mathrm{OR}=1.19,95 \% \mathrm{CI}=1.09-1.30, \mathrm{P}<0.0001)($ Table 6$)$.

\begin{tabular}{|c|c|c|c|c|c|c|c|}
\hline Study & Populations & Study stage & Case & Control & OR, Fixed, 95\% CI & OR $(95 \% \mathrm{CI})$ & Weight $\%$ \\
\hline Farrall (2006) & European & PROCARDIS & 4070 & 4258 & & $1.22(1.12-1.34)$ & 16.05 \\
\hline Clarke (2006) & European & ISIS & 2073 & 1493 & & $1.27(1.12-1.44)$ & 8.17 \\
\hline Samani (2007) & European & WTCCC & 1926 & 2937 & -1 & $1.07(0.97-1.18)$ & 13.44 \\
\hline Samani (2007) & European & GerMIFS-I & 995 & 1644 & 1 & $1.09(0.92-1.29)$ & 4.52 \\
\hline Helgadottir (2007) & European & PENN CATH & 933 & 468 & & $1.25(0.98-1.60)$ & 2.15 \\
\hline Hofman (2007) & European & Rotterdam & 779 & 5195 & + & $1.05(0.89-1.25)$ & 4.47 \\
\hline Schunkert (2008) & European & Inter Heart EW & 796 & 895 & & $1.02(0.85-1.24)$ & 3.62 \\
\hline Schunkert (2008) & Asian Indian & Inter Heart IA & 1137 & 1215 & & $1.13(0.96-1.34)$ & 4.64 \\
\hline Stirnadel (2008) & European & GEMS & 262 & 1580 & & $1.27(1.00-1.61)$ & 2.28 \\
\hline Elliott (2009) & Asian Indian & LOLIPOP IA & 1250 & 12568 & & $1.22(1.08-1.38)$ & 8.59 \\
\hline Elliott (2009) & European & LOLIPOP EW & 896 & $\mathbf{5 7 6 1}$ & & $1.16(1.02-1.32)$ & 7.77 \\
\hline Erdmann (2009) & European & GerMIFS-II & 1222 & 1298 & & $1.27(1.10-1.47)$ & 6.14 \\
\hline Stewart (2009) & European & OHS & 1542 & 1455 & & $1.43(1.25-1.64)$ & 7.00 \\
\hline Kathiresan (2009) & European & MEDSTAR & 943 & 483 & & $1.02(0.78-1.32)$ & 1.87 \\
\hline Marques (2009) & European & CoLaus & 202 & 5233 & & $0.95(0.72-1.26)$ & 1.65 \\
\hline Waterworth (2010) & European & EPIC-Norfolk1 & 421 & 2698 & & $1.22(0.99-1.50)$ & 2.99 \\
\hline Ken-Dror (2010) & European & & 275 & 2492 & & $1.09(0.84-1.41)$ & 1.92 \\
\hline Our study (2014) & Asian Chinese & & 776 & 732 & & $1.21(0.97-1.50)$ & 2.72 \\
\hline \multicolumn{3}{|l|}{ Overall } & 20498 & 52405 & 0 & $1.18(1.14-1.22)$ & 100.00 \\
\hline \multirow{2}{*}{\multicolumn{4}{|c|}{$\begin{array}{l}\text { Heterogeneity chi-squared }=\mathbf{2 4 . 4 8}(\mathrm{d} . \mathrm{f}=\mathbf{=} \mathbf{1 7}) \mathrm{P}=\mathbf{0 . 1 0 7} \\
\text { I-squared (variation in } \mathrm{ES} \text { attributable to heterogeneity) }=\mathbf{3 0 . 6 \%}\end{array}$}} & & 1.00 & & \\
\hline & & & & & & & \\
\hline
\end{tabular}

Figure 2. Meta-analysis of the studies between rs4420638 of APOE-C1-C4-C2 gene and CHD risk. WTCCC: Wellcome Trust Case Control Consortium; GerMIFS1: German MI Family Studies I; LOLIPOP EW: London Life Sciences Population European White; PROCARDIS: Precocious Coronary Artery Disease study; LOLIPOP IA: London Life Sciences Population Indian Asian; GerMIFS2: German MI Family Studies I; ISIS: International Studies of Infarct Survival; OHS: Ottawa Heart Study.

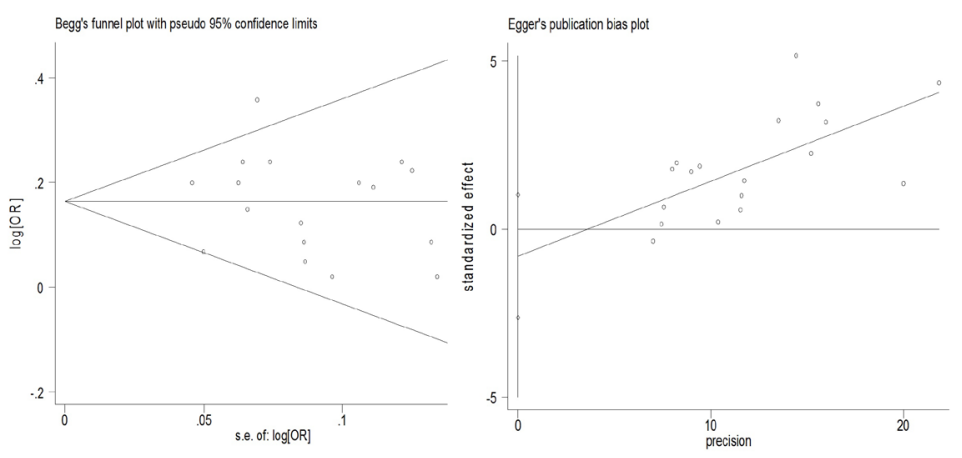

Figure 3. Funnel plot of the association between rs4420638 and CHD risk. $\mathrm{P}_{(\mathrm{Begg})}=0.13 ; \mathrm{P}_{(\mathrm{Egger})}=0.36$. 
Table 6. Summary estimates for the ORs of rs4420638 polymorphism in subgroup analyses.

\begin{tabular}{lcccccccc}
\hline Study population & Studies/stages (N) & OR $(95 \% \mathrm{CI})$ & Weight $(\%)$ & $\mathrm{Z}$ & $\mathrm{P}(\mathrm{z})$ & \multicolumn{1}{c}{$\mathrm{I}^{2}$} & $\mathrm{P}$ & Heterogeneity statistics \\
\hline European & 15 & $1.18(1.13-1.22)$ & 84.05 & 8.09 & $<0.0001$ & $41.30 \%$ & 0.048 & 23.86 \\
Asian & 3 & $1.19(1.09-1.30)$ & 15.95 & 3.82 & $<0.0001$ & $0.00 \%$ & 0.760 & 0.55 \\
Overall & 18 & $1.18(1.14-1.22)$ & 100.00 & 9.37 & $<0.0001$ & $30.60 \%$ & 0.107 & 24.48 \\
\hline
\end{tabular}

\section{DISCUSSION}

The goal of our study was to explore the association between APOE rs4420638 and circulating lipid concentrations, along with the risk of CHD in Han Chinese. Our results show that rs4420638 is significantly correlated with increased CHD risk in Han Chinese males ( $\mathrm{P}=$ $0.040, \mathrm{OR}=1.34,95 \% \mathrm{CI}=1.01-1.78)$. This variant is likely to increase the risk of CHD under the dominant model in males $(\mathrm{P}=0.036, \mathrm{OR}=1.38,95 \% \mathrm{CI}=1.02-1.88)$. Gender-specific difference has been shown in many human diseases (Weiss et al., 2006). Epidemiologic evidence shows that men have a higher cardiovascular disease risk than women worldwide (Ginter and Simko, 2013). In previous studies, we have shown that CHD risk is gender-dependent in Han Chinese (Zhou et al., 2012). This observation can possibly be explained by the hormonal profiles and behaviors that differ between the two genders (Barrett-Connor, 2007).

HDL-C, the smallest of the lipoprotein molecules, has been regarded as an antiatherogenic lipoprotein in humans (Toth, 2005). Epidemiological studies have proved that high HDL-C concentration protects against cardiovascular diseases, and low HDL-C concentrations may increase the risk of atherosclerosis (Hamrefors et al., 2010). APOA-I, the major protein component of HDL in plasma, can help clear cholesterol and contributes to atheroma regression (Asztalos and HDL Atherosclerosis Treatment Study, 2004). APOA-I protein activity is associated with high HDL-C concentration and a reduced risk of CHD (Asztalos and HDL Atherosclerosis Treatment Study, 2004). Our results also indicate that the rs4420638-AA genotype is significantly associated with the reduction of HDL-C and APOA-I levels in CHD cases.

Circulating levels of blood lipids are consistently associated with the risk of CHD (Genoux et al., 2013). The lipid levels are affected by different genetic and acquired factors. Several genes are associated with lipid levels and the risk of cardiovascular diseases (Gungor et al., 2012). APOE is a glycoprotein that plays a fundamental role in lipid metabolism. APOE polymorphisms are related to lipid levels in CHD patients (Smalinskiene et al., 2013). SNP rs4420638, located in the APOE-C1-C2 cluster, is significantly associated with plasma LDL-C and APOB concentration (Ken-Dror et al., 2010). In our study, significant interaction is found between the rs4420638 genotype and HDL-C and APOA-I concentrations in Han Chinese males. These findings could be partly explained by the particular genetic backgrounds and diet habits of the Chinese.

Several lines of study have confirmed that $A P O E$ polymorphisms contribute to the risk of CHD. A previous meta-analysis combining nine studies was performed and suggested a significant association between $A P O E$ polymorphism rs4420638 and CHD risk (Elliott et al., 2009). However, their results in Asian subgroup studies were controversial, which might have been due to the low number of studies or genetic heterogeneity. In this meta-analysis, a total of 18 studies including 72,903 individuals were examined to confirm the significant association. Our results establish that rs 4420638 results in an 18\% increase in the risk of CHD in European and Asian populations. 
There were several limitations to our study. Firstly, the sample size of the case-control study was moderate and may not have been sufficient to identify susceptible genes with moderate or minor effects. Secondly, only one SNP was checked for its association with CHD. Other genetic polymorphisms of $A P O E$ may also contribute to the risk of CHD. Further investigation in large samples is necessary for other polymorphisms that may be related to CHD in various populations. Thirdly, a power calculation for rs4420638 in the male group analysis showed that our case-control study had a $53.2 \%$ power to detect a relative risk at a significance level of 0.05 . The $P$ value was 0.040 between cases and controls in the male group, which may not have been retained after multiple test corrections. We can exclude the chance of a random positive finding of this SNP. Our sample size was comparatively small, but should have been sufficient to describe a tendency that may guide clinical practice, since our meta-analysis confirmed that rs4420638 presents a significantly increased risk of CHD in both Europeans and Asians.

In conclusion, our case-control study showed that rs4420638 in the APOE-C1-C2 cluster displays a strong relationship with CHD risk in Han Chinese males. The rs 4420638 genotype AA showed significant association with the circulating HDL-C and APOA-I concentrations in males. Meta-analysis supports the association between rs4420638 and CHD risk in European and Asian populations.

\section{Conflicts of interest}

The authors declare no conflict of interest.

\section{ACKNOWLEDGMENTS}

Research supported by grants from the Ningbo Social Development Research Projects (\#2012C50027), the Ningbo Natural Science Foundation (\#2014A610260), the Ningbo Youth and Doctor Foundation, the Ningbo High Level Innovative Talents Program, the National Natural Science Foundation of China (\#31100919 and \#81371469), the Natural Science Foundation of Zhejiang Province (\#LR13H020003), and the K.C. Wong Magna Fund in Ningbo University. The research was also supported by the Program for Professor of Special Appointments (Eastern Scholar) at Shanghai Institutions of Higher Learning, and the Key Basic Research Foundation of Science and Technology Commission of Shanghai Municipality (\#13JC1403700) (both granted to M.Q. Xu).

\section{REFERENCES}

Asztalos BF and HDL Atherosclerosis Treatment Study (2004). High-density lipoprotein metabolism and progression of atherosclerosis: new insights from the HDL Atherosclerosis Treatment Study. Curr. Opin. Cardiol. 19: 385-391.

Barrett-Connor E (2007). Commentary: Masculinity, femininity and heart disease. Int. J. Epidemiol. 36: 621-622.

Clarke R, Xu P, Bennett D, Lewington S, et al. (2006). Lymphotoxin-alpha gene and risk of myocardial infarction in 6,928 cases and 2,712 controls in the ISIS case-control study. PLoS Genet. 2: e107.

Elliott P, Chambers JC, Zhang W, Clarke R, et al. (2009). Genetic Loci associated with C-reactive protein levels and risk of coronary heart disease. JAMA 302: 37-48.

Erdmann J, Grosshennig A, Braund PS, König IR, et al. (2009). New susceptibility locus for coronary artery disease on chromosome 3q22.3. Nat. Genet. 41: 280-282.

Farrall M, Green FR, Peden JF, Olsson PG, et al. (2006). Genome-wide mapping of susceptibility to coronary artery disease identifies a novel replicated locus on chromosome 17. PLoS Genet. 2: e72. 
Genoux A, Ruidavets JB, Ferrières J, Combes G, et al. (2013). Serum IF1 concentration is independently associated to HDL levels and to coronary heart disease: the GENES study. J. Lipid Res. 54: 2550-2558.

Ginter E and Simko V (2013). Women live longer than men. Bratisl. Lek. Listy. 114: 45-49.

Graff-Iversen S, Wilsgaard T, Mathiesen EB, Njølstad I, et al. (2014). Long-term cardiovascular consequences of Rose angina at age 20-54 years: 29-years' follow-up of the Tromsø Study. J. Epidemiol. Community Health 68: 754-759.

Grallert H, Dupuis J, Bis JC, Dehghan A, et al. (2012). Eight genetic loci associated with variation in lipoprotein-associated phospholipase A2 mass and activity and coronary heart disease: meta-analysis of genome-wide association studies from five community-based studies. Eur. Heart J. 33: 238-251.

Gungor Z, Anuurad E, Enkhmaa B, Zhang W, et al. (2012). Apo E4 and lipoprotein-associated phospholipase A2 synergistically increase cardiovascular risk. Atherosclerosis 223: 230-234.

Hamrefors V, Orho-Melander M, Krauss RM, Hedblad B, et al. (2010). A gene score of nine LDL and HDL regulating genes is associated with fluvastatin-induced cholesterol changes in women. J. Lipid Res. 51: 625-634.

Heidari MM, Foruzannia SK, Khatami M, Hadadzadeh M, et al. (2013). Apolipoprotein e gene polymorphism in Iranian coronary atherosclerosis patients candidate for coronary artery bypass graft. Iran J. Basic Med. Sci. 16: 841-844.

Helgadottir A, Thorleifsson G, Manolescu A, Gretarsdottir S, et al. (2007). A common variant on chromosome 9p21 affects the risk of myocardial infarction. Science 316: 1491-1493.

Higgs ZC, Macafee DA, Braithwaite BD and Maxwell-Armstrong CA (2005). The Seldinger technique: 50 years on. Lancet 366: 1407-1409.

Hofman A, Darwish Murad S, van Duijn CM, Franco OH, et al. (2007). The Rotterdam Study: objectives and design update. Eur. J. Epidemiol. 22: 819-829.

Huang Y, Lian J, Huang RS, Wang F, et al. (2013). Positive association between rs10918859 of the NOS1AP gene and coronary heart disease in male Han Chinese. Genet. Test Mol. Biomarkers 17: 25-29.

Isley WL (2006). Low-density lipoprotein cholesterol lowering in the prevention of CHD: how low should we go? Curr. Treat. Options Cardiovasc. Med. 8: 289-297.

Jiang D, Zheng D, Wang L, Huang Y, et al. (2013). Elevated PLA2G7 gene promoter methylation as a gender-specific marker of aging increases the risk of coronary heart disease in females. PLoS One 8: e59752.

Ken-Dror G, Talmud PJ, Humphries SE and Drenos F (2010). APOE/C1/C4/C2 gene cluster genotypes, haplotypes and lipid levels in prospective coronary heart disease risk among UK healthy men. Mol. Med. 16: 389-399.

Lian J, Huang Y, Huang RS, Xu L, et al. (2013). Meta-analyses of four eosinophil related gene variants in coronary heart disease. J. Thromb. Thrombolysis 36: 394-401.

Lopes-Virella MF, Stone P, Ellis S and Colwell JA (1977). Cholesterol determination in high-density lipoproteins separated by three different methods. Clin. Chem. 23: 882-884.

Marques-Vidal P, Pécoud A, Hayoz D, Paccaud F, et al. (2009). Prevalence and characteristics of vitamin or dietary supplement users in Lausanne, Switzerland: the CoLaus study. Eur. J. Clin. Nutr. 63: 273-281.

Mendivil CO, Rimm EB, Furtado J and Sacks FM (2013). Apolipoprotein E in VLDL and LDL with apolipoprotein C-III is associated with a lower risk of coronary heart disease. J. Am. Heart Assoc. 2: e000130.

Myocardial Infarction Genetics Consortium, Kathiresan S, Voight BF, Purcell S, et al. (2009). Genome-wide association of early-onset myocardial infarction with single nucleotide polymorphisms and copy number variants. Nat. Genet. 41: 334-341.

Onat A, Can G, Murat S, Ciçek G, et al. (2013). Aggregation of lipoprotein(a) to apolipoprotein A-I underlying HDL dysfunction as a major coronary risk factor. Anadolu Kardiyol Derg. 13: 543-551.

Puddu PE, Terradura Vagnarelli O, Mancini M, Zanchetti A, et al. (2014). Typical and atypical coronary heart disease deaths and their different relationships with risk factors. The Gubbio residential cohort study. Int. J. Cardiol. 173: 300-304.

Rached FH, Chapman MJ and Kontush A (2014). An overview of the new frontiers in the treatment of atherogenic dyslipidemias. Clin. Pharmacol. Ther. 96: 57-63.

Roberts R and Stewart AF (2012). 9p21 and the genetic revolution for coronary artery disease. Clin. Chem. 58: 104-112.

Samani NJ, Erdmann J, Hall AS, Hengstenberg C, et al. (2007). Genomewide association analysis of coronary artery disease. N. Engl. J. Med. 357: 443-453.

Schunkert H, Götz A, Braund P, McGinnis R, et al. (2008). Repeated replication and a prospective meta-analysis of the association between chromosome 9p21.3 and coronary artery disease. Circulation 117: 1675-1684.

Smalinskiene A, Petkeviciene J, Luksiene D, Jureniene K, et al. (2013). Association between APOE, SCARB1, PPAR $\alpha$ polymorphisms and serum lipids in a population of Lithuanian adults. Lipids Health Dis. 12: 120.

Stewart AF, Dandona S, Chen L, Assogba O, et al. (2009). Kinesin family member 6 variant Trp719Arg does not associate with angiographically defined coronary artery disease in the Ottawa Heart Genomics Study. J. Am. Coll. Cardiol. 53: $1471-1472$. 
Stirnadel H, Lin X, Ling H, Song K, et al. (2008). Genetic and phenotypic architecture of metabolic syndrome-associated components in dyslipidemic and normolipidemic subjects: the GEMS Study. Atherosclerosis 197: 868-876.

Subedi BH, Joshi PH, Jones SR, Martin SS, et al. (2014). Current guidelines for high-density lipoprotein cholesterol in therapy and future directions. Vasc. Health Risk Manag. 10: 205-216.

Toth PP (2005). Cardiology patient page. The "good cholesterol": high-density lipoprotein. Circulation 111: e89-e91.

Waterworth DM, Ricketts SL, Song K, Chen L, et al. (2010). Genetic variants influencing circulating lipid levels and risk of coronary artery disease. Arterioscler. Thromb. Vasc. Biol. 30: 2264-2276.

Weiss LA, Pan L, Abney M and Ober C (2006). The sex-specific genetic architecture of quantitative traits in humans. Nat. Genet. 38: 218-222.

Yin YW, Sun QQ, Zhang BB, Hu AM, et al. (2013). Association between apolipoprotein E gene polymorphism and the risk of coronary artery disease in Chinese population: evidence from a meta-analysis of 40 studies. PLoS One 8: e66924.

Zhou J, Huang Y, Huang RS, Wang F, et al. (2012). A case-control study provides evidence of association for a common SNP rs974819 in PDGFD to coronary heart disease and suggests a sex-dependent effect. Thromb. Res. 130: 602-606. 